\title{
Randomized Trial of Hypofractionated, Dose-Escalated, Intensity-Modulated Radiation Therapy (IMRT) Versus Conventionally Fractionated IMRT for Localized Prostate Cancer
}

Karen E. Hoffman, K. Ranh Voong, Lawrence B. Levy, Pamela K. Allen, Seungtaek Choi, Pamela J. Schlembach, Andrew K. Lee, Sean E. McGuire, Quynh Nguyen, Thomas J. Pugh, Steven J. Frank, Rajat J. Kudchadker, Weiliang $\mathrm{Du}$, and Deborah A. Kuban

Author affiliations and support information (if applicable) appear at the end of this article.

Published at jco.org on August 14, 2018. Corresponding author: Karen E. Hoffman, MD, University of Texas MD Anderson Cancer Center, 1515 Holcombe Blvd, Unit 1202, Houston, TX 77030; e-mail: khoffman1@mdanderson.org. (C) 2018 by American Society of Clinical Oncology

$0732-183 X / 18 / 3629 w-2943 w / \$ 20.00$

\section{ASSOCIATED CONTENT}

See accompanying Oncology Grand Rounds on page 2909 Appendix

DOI: https://doi.org/10.1200/JCO 2018.77.9868

(Q) Data Supplement DOI: https://doi.org/10.1200/JCO 2018.77.9868

DOI: https://doi.org/10.1200/JCO.2018. 77.9868

\section{$\begin{array}{llllllll}\text { A } & \text { B } & \text { S } & \text { T } & \text { R } & \text { A } & \text { C } & \text { T }\end{array}$}

\section{Purpose}

Hypofractionated radiotherapy delivers larger daily doses of radiation and may increase the biologically effective dose delivered to the prostate. We conducted a randomized trial testing the hypothesis that dose-escalated, moderately hypofractionated intensity-modulated radiation therapy (HIMRT) improves prostate cancer control compared with conventionally fractionated IMRT (CIMRT) for men with localized prostate cancer.

\section{Patients and Methods}

Men were randomly assigned to $75.6 \mathrm{~Gy}$ in 1.8-Gy fractions delivered over 8.4 weeks (CIMRT) or $72 \mathrm{~Gy}$ in $2.4 \mathrm{~Gy}$ fractions delivered over 6 weeks (HIMRT, biologically equivalent to $85 \mathrm{~Gy}$ in 1.8-Gy fractions assuming prostate cancer $\alpha$-to- $\beta$ ratio of 1.5). Failure was defined as prostate-specific antigen (PSA) failure (nadir plus $2 \mathrm{ng} / \mathrm{mL}$ ) or initiation of salvage therapy. Modified Radiation Therapy Oncology Group criteria were used to grade late ( $\geq 90$ days after completion of radiotherapy) $\mathrm{Gl}$ and genitourinary toxicity.

\section{Results}

Most of the 206 men (72\%) had cT1, Gleason score 6 or 7 (99\%), and PSA level $\leq 10 \mathrm{ng} / \mathrm{mL}(90 \%)$ disease. Androgen deprivation therapy was received by $24 \%$. With a median follow-up of 8.5 years, men treated with HIMRT experienced fewer treatment failures $(\mathrm{n}=10)$ than men treated with CIMRT ( $n=21 ; P=.036)$. The 8-year failure rate was $10.7 \%(95 \% \mathrm{Cl}, 5.8 \%$ to $19.1 \%)$ with HIMRT and $15.4 \%(95 \% \mathrm{Cl}, 9.1 \%$ to $25.4 \%)$ with $\mathrm{CIMRT}$. There was no difference in overall survival $(P=$ .39). There was a nonsignificant increase in late grade 2 or $3 \mathrm{Gl}$ toxicity with HIMRT (8-year $5.0 \% \mathrm{~V}$ $12.6 \% ; P=.08)$. However, GI toxicity was only $8.6 \%$ when rectal volume receiving 65 Gy of HIMRT was $\leq 15 \%$. Late genitourinary toxicity was similar $(P=.84)$. There was no grade 4 toxicity.

\section{Conclusion}

The results of this randomized trial demonstrate superior cancer control for men with localized prostate cancer who receive dose-escalated moderately hypofractionation radiotherapy while shortening treatment duration.

\section{J Clin Oncol 36:2943-2949. (C) 2018 by American Society of Clinical Oncology}

\section{INTRODUCTION}

More than one-third of men with localized prostate cancer are treated with external-beam radiation therapy. ${ }^{1}$ Randomized trials have established that dose-escalated radiation therapy (> 75 Gy biologically equivalent dose) improves prostate cancer control and dose-escalated radiation has become standard treatment of prostate cancer. $^{2-6}$ However, it takes 8 to 9 weeks to complete dose-escalated radiation therapy when delivered with conventional fractionation (1.8 or 2.0 Gy each day Monday through Friday).

Hypofractionated prostate radiotherapy has the potential to shorten the duration of prostate cancer treatment while increasing the biologically effective dose delivered to the prostate by delivering larger daily doses of radiation. It is believed that because of differences in sensitivity to daily doses of radiation between prostate cancer 
and adjacent normal tissues, delivery of fewer, larger, daily radiation doses may increase prostate cancer control without increasing treatment adverse effects. ${ }^{7-9}$ Shorter hypofractionated treatment is also more convenient for patients, decreases the cost of treatment, and may increase patient access to treatment. ${ }^{10-12}$

A randomized trial was undertaken to test the hypothesis that shorter-duration, moderately hypofractionated, intensitymodulated radiation therapy (HIMRT; 72 Gy in 2.4-Gy fractions delivered over 6 weeks) improves prostate cancer control without increasing treatment toxicity.

\section{PATIENTS AND METHODS}

Men with organ-confined prostate cancer provided informed consent to enroll in a phase III institutional review board-approved trial from January 2001 to January 2010 that randomly assigned men to either a conventional fractionation intensity-modulated radiation therapy (CIMRT) regimen that delivered 75.6 Gy in 1.8-Gy fractions or to a dose-escalated moderate HIMRT regimen that delivered 72 Gy in 2.4-Gy fractions (ClinicalTrials. gov identifier: NCT00667888). The moderate hypofractionation regimen was biologically equivalent to delivering 85 Gy in 1.8 -Gy fractions, assuming an $\alpha$-to- $\beta$ ratio of 1.5 Gy for prostate cancer. Therefore, the hypofractionation regimen delivered a higher biologically effective dose. The dose escalation difference was designed to be similar to the MD Anderson trial that demonstrated improved prostate cancer control with conventionally fractionated dose escalation. ${ }^{5}$

\section{Eligibility Criteria}

Eligible patients had biopsy-proven prostate adenocarcinoma, good performance status (Zubrod $<2$ ), clinical tumor T1b to T3b disease (1992 American Joint Committee on Cancer staging system ${ }^{13}$ ), PSA level $\leq 20$ $\mathrm{ng} / \mathrm{mL}$, Gleason score $<10$, and no clinical, radiographic or pathologic evidence of nodal or bone metastasis. Bone scan within 3 months of signing protocol consent or starting androgen deprivation therapy (ADT) was required for men with PSA levels $>10 \mathrm{ng} / \mathrm{mL}$ or cT3 disease, and pelvic CT scan was required for men with cT3 disease. Men with cT3 disease were required to have a Gleason score $<8$ and pretreatment PSA level $\leq 10 \mathrm{ng} / \mathrm{mL}$. Men with Gleason-score- 8 - or- 9 disease were required to have $\mathrm{cT} 1 / \mathrm{T} 2$ disease and pretreatment PSA levels $\leq 10 \mathrm{ng} / \mathrm{mL}$. Men were not eligible for enrollment if they had palpable stage cT3c (seminal vesicle involvement ${ }^{13}$ ) or cT4 disease; a history of prior pelvic radiotherapy; received $>4$ months of ADT; prior or planned radical prostate surgery; or if they had concurrent active malignancy other than nonmetastatic skin cancer or early stage chronic lymphocytic leukemia. Men were stratified at randomization by PSA level $(\leq 10 v>10 \mathrm{ng} / \mathrm{mL})$, stage (cT1b-T2 $v$ cT3ab), Gleason score ( $\leq 7 v 8$ to 9 ), and receipt of neoadjuvant ADT (yes $v$ no).

\section{Radiation Therapy}

All men were treated with step-and-shoot IMRT, as previously described. ${ }^{14}$ Daily prostate localization for treatment alignment was primarily performed using BAT ultrasound (Best Nomos, Pittsburgh, PA); daily kilovoltage imaging with fiducial alignment was permitted in the later years of the trial. The treatment target was the prostate and proximal seminal vesicles. Lymph nodes were not treated. Per protocol, planning target volume (PTV) expansion was 10 to $15 \mathrm{~mm}$ in all dimensions except for 4 to $8 \mathrm{~mm}$ posteriorly. The protocol stipulated that $\geq 95 \%$ of the PTV receive the prescription dose and that inhomogeneity be $\leq 20 \%$. The rectum was defined as the portion of bowel extending from the sigmoid flexure to the pelvic outlet. The protocol recommended that for men treated with conventional fractionation, the proportion of rectum and bladder receiving 70 Gy be $<20 \%$ to $25 \%$ and, for men treated with hypofractionation, the proportion of rectum and bladder receiving 65 Gy be $<20 \%$ to $25 \%$. These recommended constraints were not met in some patients (Appendix Table A1, online only).

\section{Follow-Up}

After completion of radiotherapy, men were seen at least every 6 months for the first 2 years and then annually thereafter. PSA was obtained every 3 months for the first 2 years, every 6 months for years 3 through 5 , and then yearly thereafter. Physician-reported toxicity was evaluated at each follow-up visit. Late genitourinary (GU) and GI toxicity, starting 90 days after completion of radiotherapy, was quantified using the modified Radiation Therapy Oncology Group (RTOG) toxicity grading. ${ }^{14,15}$

\section{Statistical analysis}

Men were classified into risk groups based on National Comprehensive Cancer Network criteria (low: cT stage $\leq \mathrm{T} 2 \mathrm{a}$, Gleason score $\leq 6$, and PSA level $<10 \mathrm{ng} / \mathrm{mL}$; high: cT stage $>\mathrm{T} 2 \mathrm{c}$, or Gleason score $\geq 8$, or PSA level $>20 \mathrm{ng} / \mathrm{mL}$; intermediate: all others). ${ }^{16}$ Descriptive statistics were generated to characterize the study cohort. Characteristics were compared with the $\chi^{2}$ test or Fisher exact test for categorical variables and the Wilcoxon rank-sum test or Student $t$ test for continuous variables. The primary outcome was failure, defined as PSA failure or initiation of salvage therapy. PSA failure was defined using the Phoenix definition of PSA nadir plus $2 \mathrm{ng} / \mathrm{mL} .{ }^{17}$ The Phoenix definition, developed in 2005, is the current standard definition for biochemical failure after radiation therapy.

Time to failure was calculated from the start of radiation therapy. Time to grade 2 or higher GU or GI toxicity was measured from the end of radiation therapy. Kaplan-Meier curves were created to estimate failure, survival, and toxicity. ${ }^{18}$ A log-rank test evaluated the estimates across strata. $\mathrm{P}<.05$ was considered statistically significant. Data analysis was performed using Stata/MP 15.0 statistical software (StataCorp, College Station, TX). ${ }^{19}$

As-treated analyses were performed because this approach best represents the toxicity and cancer control from the radiation treatment regimens. We also report an intent-to-treat analysis for the primary end point, treatment failure.

\section{Sample Size and Previous Analyses}

At time of protocol design, it was estimated $30 \%$ of men treated with conventional fractionation and $10 \%$ of men treated with hypofractionation would develop PSA failure at 5 years. Based on log-rank test results, a sample size of 226 was needed to ensure at least $91 \%$ statistical power to detect a $20 \%$ difference in freedom from failure at 5 years at the two-sided 0.05 significance level. Thirty-nine events were anticipated based on this power calculation.

The study was monitored by a Data Safety Monitoring Board and the stopping rule for toxicity was never met. There were too few events during accrual to justify interim analysis of efficacy. We previously published patient-reported outcomes, toxicity predictors, and treatment costs. ${ }^{12,14,20}$ A preliminary analysis of cancer control was presented with a median follow-up 4.7 years, at which time there were only 16 failure events. ${ }^{21}$

\section{RESULTS}

\section{Study Cohort}

In each treatment group (ie, conventional fractionation and hypofractionation), 102 of 111 men completed treatment as planned. One man allocated to 75.6 Gy actually received 72 Gy and one man allocated to 72 Gy died during treatment of a nontreatment-, noncancer-related cause. For this as-treated analysis, the 102 men who received conventional radiotherapy were compared with the 104 men who initiated or received hypofractionated radiotherapy (Fig 1).

Most men had T1 disease (72\%), Gleason score 7 disease (65\%), PSA level $\leq 10 \mathrm{ng} / \mathrm{mL}$ (90\%), and did not receive ADT (76\%). 


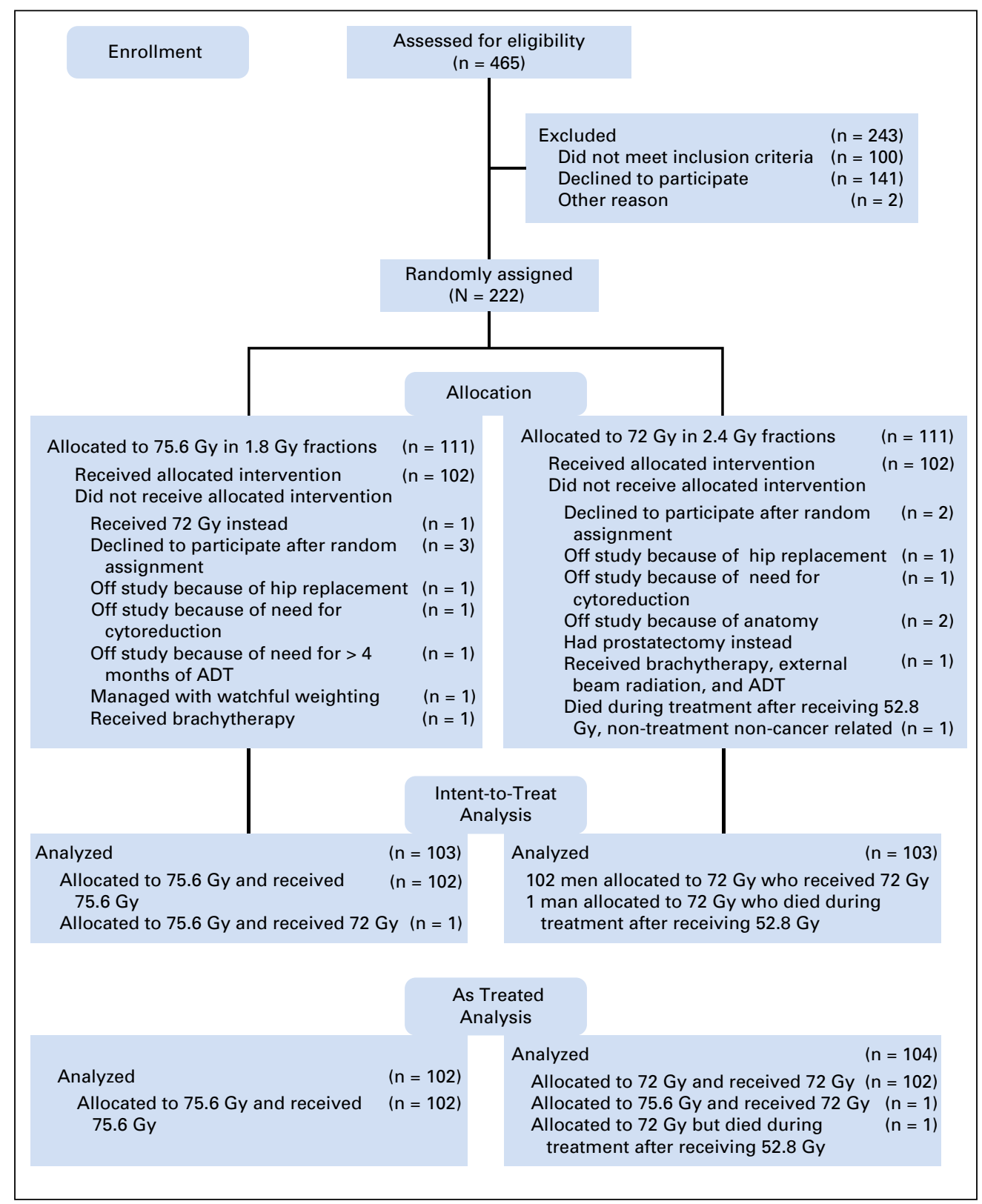

Fig 1. CONSORT diagram. ADT, androgen deprivation therapy; CIMRT, conventional intensity modulated radiation therapy; HIMRT, hypofractionated intensity modulated radiation therapy; RT, radiation therapy.

Distribution of patient demographics and clinical prognostic factors was well-balanced between treatment groups (Table 1).

\section{Treatment Failure}

Median follow-up at time of analysis was 8.5 (range, 0.1 to 14.5) years. Thirty-one men developed treatment failure (treated with hypofractionation $[\mathrm{n}=10]$, treated with conventional fractionation $[n=21])$. Twenty-eight of these men had PSA failure and three received salvage therapy with initiation of ADT. Men who received hypofractionation were less likely to develop failure $(P=$ .036; Fig 2A). Five-year, 8-year, and 10-year cumulative incidence of failure was $8.2 \%$ (95\% CI, $4.2 \%$ to $15.8 \%), 10.7 \%$ (95\% CI, $5.9 \%$ to $19.1 \%$ ), and $10.7 \%$ (95\% CI, $5.9 \%$ to $19.1 \%)$, respectively, for men treated with hypofractionation. Five-year, 8 -year, and 10year cumulative incidence of failure was $8.3 \%$ (95\% CI, $4.2 \%$ to
$15.9 \%$ ), $15.4 \%$ (95\% CI, $9.1 \%$ to $25.4 \%$ ), and $23.7 \%$ (95\% CI, $15.0 \%$ to $36.3 \%$ ), respectively, for men treated with conventional fractionation. When time-to-failure was evaluated in an intent-totreat analysis, men randomly assigned to hypofractionated treatment were also less likely to develop failure $(P=.01)$.

Because men were stratified at randomization by receipt of ADT and PSA level $\leq 10 \mathrm{ng} / \mathrm{mL}$, we evaluated failure for these subgroups. Failure events and cumulative incidence of failure are summarized in Appendix Tables A2 and A3. Among the 156 men who did not receive ADT, men who received hypofractionation were less likely to develop failure $(P=.033$; Fig $2 \mathrm{~B})$. Eight-year cumulative incidence of failure was $12.7 \%(95 \% \mathrm{CI}, 6.8 \%$ to $23.0 \%$ ) for men treated with hypofractionation and $18.0 \%$ (95\% CI, $10.5 \%$ to $29.8 \%$ ) for men treated with conventional fractionation. Among the 50 men who received ADT, there was no difference in failure between treatment groups (hypofractionation 


\begin{tabular}{|c|c|c|}
\hline Characteristic & $\begin{array}{l}\text { Hypofractionation } \\
\text { (72 Gy in } 2.4 \mathrm{~Gy} \\
\text { fractions) }\end{array}$ & $\begin{array}{l}\text { Conventional } \\
\text { Fractionation } \\
(75.6 \mathrm{~Gy} \text { in } 1.8 \mathrm{~Gy} \\
\text { fractions) }\end{array}$ \\
\hline No. of men & 104 & 102 \\
\hline \multicolumn{3}{|l|}{ Age, years } \\
\hline$<60$ & $15(14.4)$ & $14(13.7)$ \\
\hline $60-54$ & $17(16.4)$ & $28(27.5)$ \\
\hline $65-69$ & $26(25.0)$ & $24(23.5)$ \\
\hline$\geq 70$ & $46(44.2)$ & 36 (35.3) \\
\hline \multicolumn{3}{|l|}{ Race } \\
\hline Asian & $5(4.8)$ & $6(5.9)$ \\
\hline Black & $13(12.5)$ & $17(16.7)$ \\
\hline Hispanic & $7(6.7)$ & $8(7.8)$ \\
\hline White & $79(76)$ & $70(68.6)$ \\
\hline Other & $0(0)$ & $1(1.0)$ \\
\hline \multicolumn{3}{|l|}{ Tumor stage } \\
\hline $\mathrm{T} 1 \mathrm{~b}$ & $0(0)$ & $1(1.0)$ \\
\hline T1c & 71 (68.3) & 76 (74.5) \\
\hline T2 & $1(1.0)$ & $0(0)$ \\
\hline $\mathrm{T} 2 \mathrm{a}$ & $25(24.0)$ & $17(16.7)$ \\
\hline $\mathrm{T} 2 \mathrm{~b}$ & $4(3.9)$ & 7 (6.9) \\
\hline T2c & $3(2.9)$ & $1(1.0)$ \\
\hline \multicolumn{3}{|c|}{ PSA concentration, $\mathrm{ng} / \mathrm{mL}$} \\
\hline $0-10$ & $95(91.4)$ & $91(89.2)$ \\
\hline $10.1-20$ & $9(8.6)$ & $11(10.8)$ \\
\hline \multicolumn{3}{|l|}{ Gleason score } \\
\hline 6 & 33 (31.7) & 37 (36.3) \\
\hline 7 & $70(67.3)$ & $64(62.7)$ \\
\hline 8 & $1(1.0)$ & $1(1.0)$ \\
\hline \multicolumn{3}{|l|}{ NCCN risk-group } \\
\hline Low & 28 (26.9) & $29(28.4)$ \\
\hline Intermediate & $75(72.1)$ & 72 (70.6) \\
\hline High & $1(1.0)$ & $1(1.0)$ \\
\hline \multicolumn{3}{|l|}{ ADT } \\
\hline Yes ( $\leq 4$ months) & $27(26.0)$ & $23(22.5)$ \\
\hline No & 77 (74.0) & 79 (77.5) \\
\hline \multicolumn{3}{|c|}{$\begin{array}{l}\text { NOTE. Data given as No. (\%) unless otherwise indicated. There were } n \\
\text { significant between-arm differences. All } P \text { values }>.05 \text {. } \\
\text { Abbreviations: ADT, androgen deprivation therapy; NCCN, National Compre } \\
\text { hensive Cancer Network; PSA, prostate-specific antigen. } \\
\text { *The treatment regimen for the as-treated analysis. }\end{array}$} \\
\hline
\end{tabular}

arm, 8-year cumulative incidence: $4.2 \%$ [ $95 \% \mathrm{CI}, 1.0 \%$ to $26.1 \%$ ] $v$ $4.5 \%$ [ $95 \% \mathrm{CI}, 1.0 \%$ to $28.1 \%$ ]; $P=.94$ ).

Among the 186 men with PSA $\leq 10 \mathrm{ng} / \mathrm{mL}$, there were fewer failures among men treated with hypofractionation (hypofractionation arm, 8-year cumulative incidence: $10.5 \%$ [95\% CI, 5.6\% to $19.3 \%$ ], $P=.042 v$ conventional fractionation arm, 8 -year cumulative incidence: $14.8 \%$ [95\% CI, $8.3 \%$ to $25.6 \%$ ]; Fig 2C; Appendix Table A3, online only). Among the 20 men with PSA $>10 \mathrm{ng} / \mathrm{mL}$, there was no difference in failure between treatment groups $(P=.62)$.

\section{Prostate Cancer and Overall Survival}

There was only one clinically apparent failure; one man treated with hypofractionation developed distant metastasis. Of the 43 men who died, 19 were treated with hypofractionation and 24 were treated with conventional fractionation. No one died of prostate cancer. There was no difference in overall survival between treatment groups $(P=.39$; Fig 3). Eight- and 10-year overall survival was $90.0 \%$ ( $95 \% \mathrm{CI}, 82.2 \%$ to $94.5 \%)$ and $82.8 \%$ (95\% CI, $72.0 \%$ to $89.8 \%$ ), respectively, for men treated with hypofractionation. Eight- and 10-year overall survival was $85.2 \%$ (95\%
CI, $76.2 \%$ to $91.0 \%$ ) and $76.1 \%$ (95\% CI, $64.3 \%$ to $84.4 \%$ ), respectively, for men treated with conventional fractionation.

\section{Late GI and GU Toxicity}

Risk of late GI toxicity was similar between treatment groups. The 8-year cumulative incidence of grade 2 or 3 late GI toxicity was $5.0 \%$ (95\% CI, $2.11 \%$ to $11.6 \%)$ in men treated with conventional fractionation and $12.6 \%(95 \% \mathrm{CI}, 7.3 \%$ to $21.2 \%)$ in men treated with hypofractionation $(P=.08$; Fig $4 \mathrm{~A})$. In the conventional arm, 17 men (17\%) developed grade 1 GI toxicity, four men (4\%) developed grade 2 GI toxicity, and one man (1\%) developed grade 3 GI toxicity. In the hypofractionated arm, 26 men $(25 \%)$ developed grade 1 GI toxicity, 10 men (10\%) developed grade 2 GI toxicity, and two men (2\%) developed grade 3 GI toxicity. Nearly all grade 2 toxicities were the result of rectal bleeding (13 of 14 cases). All grade 3 toxicities were the result of persistent rectal bleeding that required two or more cauterizations (three of three cases). Neither treatment group had any grade 4 GI toxicity events.

Risk of late GU toxicity was similar between treatment groups. The 8-year cumulative incidence of grade 2 or 3 late GU toxicity was $16.4 \%$ (95\% CI, $10.4 \%$ to $25.4 \%$ ) in the conventional arm and $15.1 \%$ (95\% CI, 9.4\% to $23.8 \%)$ in the hypofractionated arm $(P=.84$; Fig $4 \mathrm{~B})$. In the conventional arm, 14 men (14\%) developed grade $1 \mathrm{GU}$ toxicity, 15 men (15\%) developed grade $2 \mathrm{GU}$ toxicity, and one man (1\%) developed grade $3 \mathrm{GU}$ toxicity. In the hypofractionated arm, 10 men (10\%) developed grade 1 GU toxicity, 15 men (15\%) developed grade 2 GU toxicity, and no men ( $0 \%$ ) developed grade 3 GU toxicity. Neither treatment group had any grade 4 GU toxicity events.

\section{Effect of Rectal Dose on Late GI Toxicity}

In a prior analysis, we determined the risk of late GI toxicity after this hypofractionation regimen could be reduced by minimizing the proportion of rectum receiving high-dose radiation. ${ }^{14}$ Now with longer follow-up, we evaluated the risk of developing grade 2 or higher late GI toxicity when the volume of rectum receiving $65 \mathrm{~Gy}$ was $\leq 15 \%$. This cutoff was selected because it is the constraint we currently use in our clinical practice with this regimen. The 8-year cumulative incidence of grade 2 or 3 late GI toxicity after hypofractionation was $8.6 \%$ (95\% CI, 3.6\% to $19.9 \%$ ) when V65 was $\leq 15 \%$, and incidence was $20.5 \%$ (95\% CI, $10.3 \%$ to $38.3 \%)$ when V65 was $>15 \%(P=.061$; Appendix Fig A1).

\section{DISCUSSION}

In this randomized trial with a median follow-up of 8.5 years, a moderately hypofractionated, dose-escalated, IMRT regimen (72 Gy in 2.4-Gy fractions) that administered a higher biologically effective radiation dose to the prostate provided superior prostate cancer control than a conventionally fractionated IMRT regimen (75.6 Gy in 1.8-Gy fractions) while shortening treatment duration from 8.4 weeks to 6 weeks. Although the men in the dose-escalated hypofractionated regimen arm received larger daily doses of radiation, the treatment did not increase incidence of late urinary toxicity. There was a nonsignificant increase in rectal bleeding after radiation for men treated with hypofractionation; all cases resolved with treatment. 


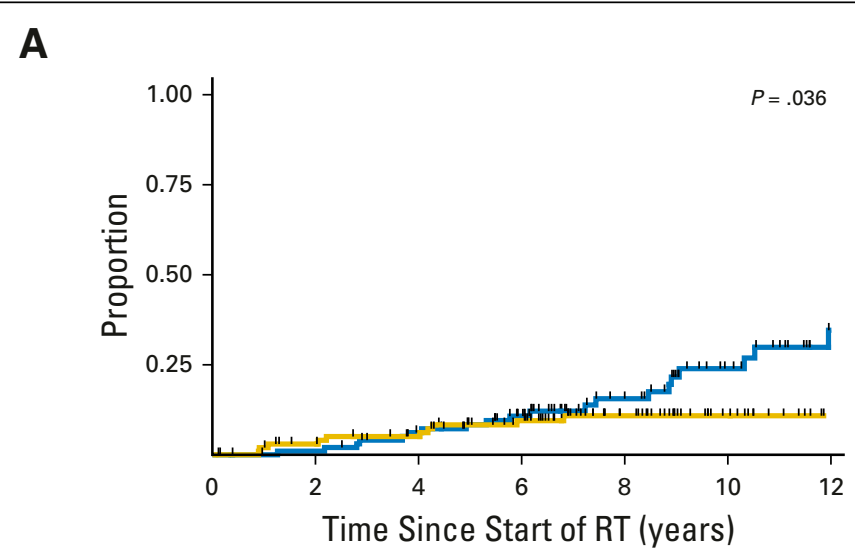

No. at risk:

\begin{tabular}{rrrrrrrr} 
CIMRT & 102 & 99 & 89 & 69 & 47 & 28 & 13 \\
\hline HIMRT & 104 & 95 & 89 & 79 & 53 & 33 & 19
\end{tabular}

C

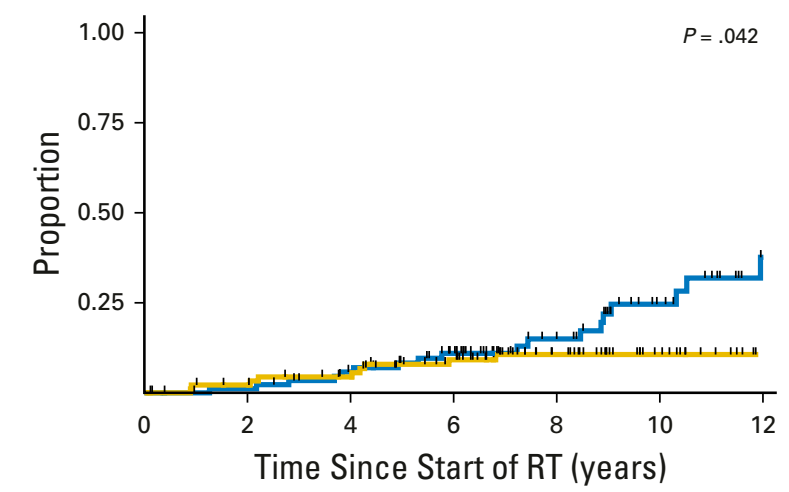

No. at risk:

$\begin{array}{llllllll}\text { CIMRT } & 91 & 88 & 79 & 61 & 41 & 23 & 10\end{array}$
B

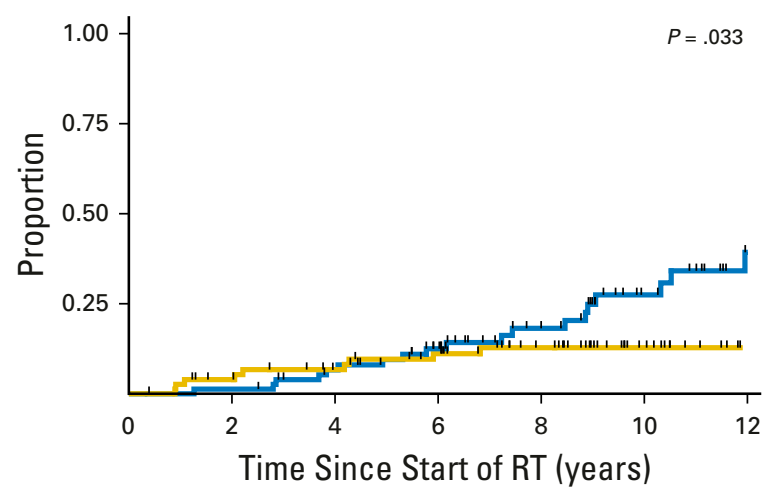

No. at risk:

$\begin{array}{rrrrrrrr}\text { CIMRT } & 79 & 77 & 68 & 55 & 40 & 23 & 11 \\ \text { HIMRT } & 77 & 71 & 65 & 59 & 47 & 30 & 17\end{array}$

Fig 2. Cumulative incidence of treatment failure after CIMRT or HIMRT for (A) all patients, (B) men who did not receive androgen deprivation therapy, and (C) men with

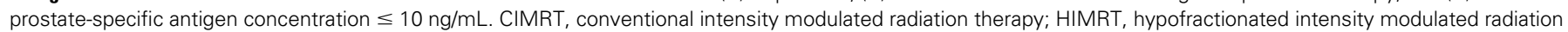
therapy; RT, radiation therapy.

This randomized trial reports superior prostate cancer control after moderately hypofractionated, dose-escalated, IMRT. The improved cancer control is the consequence of delivery of a higher biologically effective dose to the prostate in the hypofractionated treatment arm (ie, 85 Gy equivalent in 1.8-Gy fractions assuming an $\alpha$-to- $\beta$ ratio of 1.5 for prostate cancer). Superior cancer control with dose-escalated radiation has been established in randomized trials using conventionally fractionated radiation and dose-escalated radiation therapy is now standard treatment for men who receive prostate radiation therapy. ${ }^{2-6,29}$ However, it takes 8 to 9 weeks to deliver doseescalated radiation when using conventional fractionation. In contrast, this moderately hypofractionated radiation treatment regimen was completed in 6 weeks. Shorter-duration radiation treatment reduces a patient's time away from personal, work, and family obligations and reduces out-of-pocket expenses. Shortening treatment duration from 42 treatments to 30 treatments also reduces the cost of treatment as it is currently reimbursed in the United States, because the cost of radiation treatment is proportional to the number of treatments delivered.

This trial demonstrated improved prostate cancer control with dose-escalated moderately hypofractionated IMRT because of the length of follow-up. In this trial, the prostate cancer control curves began to separate after 5 years and, to date, the other published randomized trials in which dose-escalated moderate hypofractionation was evaluated have reported 5-year results, including Radiation Therapy Oncology Group (RTOG) 0415, the Hypofractionated Irradiation for Prostate Cancer trial (HYPRO), and the Fox Chase trial (ClinicalTrials.gov identifier: NCT00062309). ${ }^{22-24}$

In addition, a larger portion of men in the HYPRO and Fox Chase trials received ADT, which could mask the effect of dose escalation on cancer control. The Conventional or Hypofractionated High-Dose Intensity Modulated Radiotherapy in Prostate Cancer (CHHiP) trial and the Prostate Fractionated Irradiation Trial (PROFIT) also reported similar cancer control at 5 years but evaluated moderately hypofractionated radiation regimens calculated to deliver a dose equivalent to the conventionally fractionated treatment arm. ${ }^{25,26}$

This moderately hypofractionation regimen did not increase late urinary toxicity and had only a modest effect on late GI toxicity. This is consistent with other randomized trials comparing moderately hypofractionated regimens (2.4 to 3.0 Gy per treatment) with conventionally fractionated regimens that have demonstrated either 


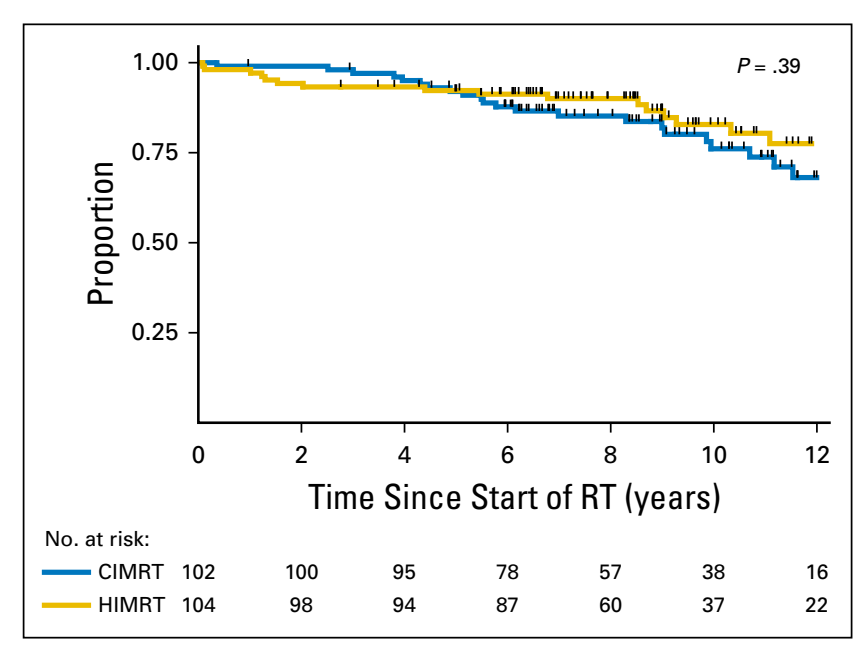

Fig 3. Overall survival for men who received CIMRT or HIMRT. CIMRT, conventional intensity modulated radiation therapy; HIMRT, hypofractionated intensity modulated radiation therapy; $\mathrm{RT}$, radiation therapy.

similar late GU and GI toxicity with moderate hypofractionation (CHHiP, PROFIT) or a small increase (RTOG 0415). ${ }^{25-27}$

We previously reported the risk of developing late GI toxicity after this moderately hypofractionated regimen can be reduced by minimizing the proportion of rectum receiving moderate- and high-dose radiation. ${ }^{14}$ Now with a median follow-up of 8.5 years, the cumulative incidence of late grade 2 or 3 GI toxicity was $<10 \%$, when the volume of rectum receiving 65 Gy was $\leq 15 \%$. This underscores the importance of adherence to normal tissue-dose constraints, especially rectal dose constraints, to limit toxicity after treatment with moderately hypofractionated prostate radiation.

It should be noted that this trial was conducted in the early days of IMRT and image-guided radiation therapy. Delivery of contemporary radiotherapy that uses smaller PTV expansions has the ability to reduce dose delivered to the rectum and bladder and may produce better toxicity outcomes. Physician-assessed rather than patient-reported toxicity outcomes are reported and patient report can differ from physician assessment. ${ }^{28}$ We previously reported no detectable differences in patient-reported urinary, bowel, and sexual symptom burden between men in the treatment arms in this trial. ${ }^{20}$ The RTOG 0415 and CHHiP trials also had similar patient-reported outcomes across treatment arms. ${ }^{26,27}$

The strength of this study is the long follow-up of $>8$ years. The 75.6-Gy conventional fractionation regimen evaluated in this trial is still a standard treatment regimen for prostate cancer. ${ }^{29}$ The $\alpha$-to- $\beta$ ratio of prostate cancer is an estimate and it may actually be lower or higher than the $\alpha$-to- $\beta$ ratio of 1.5 used to calculate the biologically equivalent dose of 85 Gy in 1.8-Gy fractions for the moderate hypofractionation arm. Regardless of the actual $\alpha$-to- $\beta$ ratio for prostate cancer, this moderately hypofractionated regimen shortened the duration of treatment while improving cancer control. The rate of prostate cancer failure after treatment was lower than anticipated at time of trial development; therefore, it has taken longer than initially anticipated to develop enough prostate cancer failure events to evaluate a difference between treatment arms. We postulate that men enrolled in the trial had more favorable disease than the historical cohort used to estimate expected failure, because of stage migration and exclusion of men receiving $>4$ months of ADT. There were a limited number of events for the subgroup analyses, but the results of the majority subgroups are consistent with the overall trial results. It took longer than anticipated to accrue patients because of competing treatments that gained enthusiasm during the study period, including robotic surgery, proton radiation, and the administration of $>4$ months of ADT. The results of this study are most applicable to men with low-risk and intermediate-risk prostate cancer, because only two men with high-risk prostate cancer were enrolled in this trial. Similarly, the toxicity results are most applicable to men receiving IMRT delivered with image-guided radiation therapy targeting the prostate and proximal seminal vesicles.

In conclusion, compared with CIMRT (75.6 in 1.8-Gy fractions), this dose-escalated moderate HIMRT regimen (72 Gy in 2.4-Gy fractions) shortened treatment duration while improving prostate cancer control. GU toxicity was similar. Although there was a nonsignificant increase in late grade 2 and 3 GI toxicity with

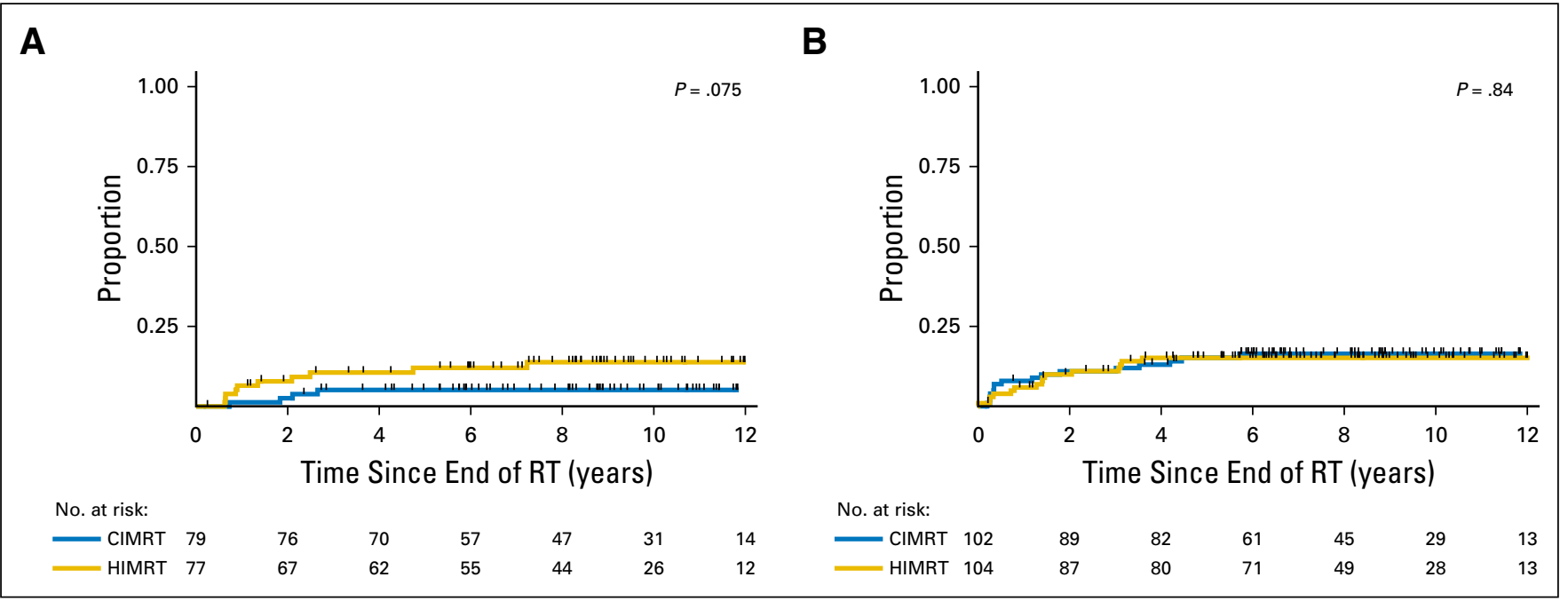

Fig 4. (A) Grade 2 or 3 late GI (grade 2a) and (B) genitourinary (grade 2b) toxicity in men who received CIMRT or HIMRT. CIMRT, conventional intensity modulated radiation therapy; HIMRT, hypofractionated intensity modulated radiation therapy; $\mathrm{RT}$, radiation therapy. 
dose-escalated hypofractionated treatment, the number of men who developed toxicity was small, all rectal bleeding resolved with treatment, and the risk of bleeding can be reduced by minimizing the proportion of rectum that receives high-dose radiation.

\section{AUTHORS' DISCLOSURES OF POTENTIAL CONFLICTS} OF INTEREST

Disclosures provided by the authors are available with this article at jco.org.

\section{AUTHOR CONTRIBUTIONS}

Conception and design: Deborah A. Kuban

Provision of study materials or patients: Pamela K. Allen, Seungtaek Choi, Andrew K. Lee, Deborah A. Kuban

Collection and assembly of data: Karen E. Hoffman, Lawrence B. Levy, Deborah A. Kuban

Data analysis and interpretation: All authors

Manuscript writing: All authors

Final approval of manuscript: All authors

Accountable for all aspects of the work: All authors

\section{REFERENCES}

1. Mahmood U, Levy LB, Nguyen $P L$, et al: Current clinical presentation and treatment of localized prostate cancer in the United States. J Urol 192: 1650-1656, 2014

2. Beckendorf $\mathrm{V}$, Guerif $\mathrm{S}$, Le Prisé $\mathrm{E}$, et al: $70 \mathrm{~Gy}$ versus $80 \mathrm{~Gy}$ in localized prostate cancer: 5 -Year results of GETUG 06 randomized trial. Int J Radiat Oncol Biol Phys 80:1056-1063, 2011

3. Dearnaley DP, Jovic G, Syndikus I, et al: Escalated-dose versus control-dose conformal radiotherapy for prostate cancer: Long-term results from the MRC RT01 randomised controlled trial. Lancet Oncol 15:464-473, 2014

4. Heemsbergen WD, Al-Mamgani $A$, Slot $A$, et al: Long-term results of the Dutch randomized prostate cancer trial: impact of dose-escalation on local, biochemical, clinical failure, and survival. Radiother Oncol 110:104-109, 2014

5. Kuban DA, Tucker SL, Dong L, et al: Long-term results of the M. D. Anderson randomized doseescalation trial for prostate cancer. Int J Radiat Oncol Biol Phys 70:67-74, 2008

6. Zietman $A L$, Bae $K$, Slater JD, et al: Randomized trial comparing conventional-dose with highdose conformal radiation therapy in early-stage adenocarcinoma of the prostate: Long-term results from Proton Radiation Oncology Group/American College of Radiology 95-09. J Clin Oncol 28:1106-1111, 2010

7. Brenner DJ, Martinez AA, Edmundson GK, et al: Direct evidence that prostate tumors show high sensitivity to fractionation (low alpha/beta ratio), similar to late-responding normal tissue. Int J Radiat Oncol Biol Phys 52:6-13, 2002

8. Fowler J, Chappell R, Ritter M: Is alpha/beta for prostate tumors really low? Int J Radiat Oncol Biol Phys 50:1021-1031, 2001

9. King CR, Fowler JF: A simple analytic derivation suggests that prostate cancer alpha/beta ratio is low. Int J Radiat Oncol Biol Phys 51:213-214, 2001

10. Konski AA: Less is more: Will hypofractionated radiotherapy negatively affect cancer centers or be a Godsend in the new health care environment? Oncology (Williston Park) 26:522-523, 2012

11. Sethukavalan $\mathrm{P}$, Cheung $\mathrm{P}$, Tang $\mathrm{Cl}$, et al: Patient costs associated with external beam radiotherapy treatment for localized prostate cancer: The benefits of hypofractionated over conventionally fractionated radiotherapy. Can J Urol 19:6165-6169, 2012

12. Voong KR, Lal LS, Kuban DA, et al: Long-term economic value of hypofractionated prostate radiation: Secondary analysis of a randomized trial. Adv Radiat Oncol 2:249-258, 2017

13. Beahrs $\mathrm{OH}$, Henson DE, Hutter RVP, et al (eds): American Joint Committee on Cancer. Manual for Staging of Cancer, 4th ed. Philadelphia, PA, JB Lippincott Co., 1992

14. Hoffman KE, Voong KR, Pugh $T J$, et al: Risk of late toxicity in men receiving dose-escalated hypofractionated intensity modulated prostate radiation therapy: Results from a randomized trial. Int J Radiat Oncol Biol Phys 88:1074-1084, 2014

15. Cox JD, Stetz J, Pajak TF: Toxicity criteria of the Radiation Therapy Oncology Group (RTOG) and the European Organization for Research and Treatment of Cancer (EORTC). Int J Radiat Oncol Biol Phys 31:1341-1346, 1995

16. NCCN Clinical Practice Guidelines in Oncology: Prostate cancer. Version 1.2015. www.nccn. org/professionals/physician_gls/pdf/prostate.pdf.

17. Roach M III, Hanks G, Thames H, Jr., et al: Defining biochemical failure following radiotherapy with or without hormonal therapy in men with clinically localized prostate cancer: Recommendations of the RTOG-ASTRO Phoenix Consensus Conference. Int J Radiat Oncol Biol Phys 65: 965-974, 2006

18. Kaplan EL, Meier P: Nonparametric estimation from incomplete observations. J Am Stat Assoc 53: 457-481, 1958

19. StataCorp. Stata: Release 15. Statistical Software. College Station, TX, StataCorp LP, 2017.

20. Hoffman KE, Skinner H, Pugh TJ, et al: Patientreported urinary, bowel, and sexual function after hypofractionated intensity-modulated radiation therapy for prostate cancer: Results from a randomized trial. Am J Clin Oncol, 41:558-567, 2018

21. Kuban DA, Nogueras-Gonzalez GM, Hamblin L, et al: Preliminary report of a randomized dose escalation trial for prostate cancer using hypofractionation. Int J Radiat Oncol Biol Phys 78:S58-S59, 2010

22. Incrocci L, Wortel RC, Alemayehu WG, et al: Hypofractionated versus conventionally fractionated radiotherapy for patients with localised prostate cancer (HYPRO): Final efficacy results from a randomised, multicentre, open-label, phase 3 trial. Lancet Oncol 17:1061-1069, 2016

23. Lee WR: Prostate cancer and the hypofractionation hypothesis. J Clin Oncol 31:3849-3851, 2013

24. Pollack A, Walker G, Horwitz EM, et al: Randomized trial of hypofractionated external-beam radiotherapy for prostate cancer. J Clin Oncol 31: 3860-3868, 2013

25. Dearnaley D, Syndikus I, Mossop H, et al: Conventional versus hypofractionated high-dose intensity-modulated radiotherapy for prostate cancer: 5-Year outcomes of the randomised, noninferiority, phase $3 \mathrm{CHHiP}$ trial. Lancet Oncol 17: 1047-1060, 2016

26. Catton CN, Lukka H, Gu CS, et al: Randomized trial of a hypofractionated radiation regimen for the treatment of localized prostate cancer. J Clin Oncol 35:1884-1890, 2017

27. Lee WR, Dignam JJ, Amin MB, et al: Randomized phase III noninferiority study comparing two radiotherapy fractionation schedules in patients with low-risk prostate cancer. J Clin Oncol 34:2325-2332, 2016

28. Watkins-Bruner $D$, Scott $C$, Lawton $C$, et al: RTOG's first quality of life study-RTOG 90-20: A phase II trial of external beam radiation with etanidazole for locally advanced prostate cancer. Int J Radiat Oncol Biol Phys 33:901-906, 1995

29. NCCN Clinical Practice Guidelines in Oncology. Prostate cancer. Version 2.2018. www.nccn. org/professionals/physician_gls/pdf/prostate.pdf.

\section{Affiliations}

Karen E. Hoffman, Lawrence B. Levy, Pamela K. Allen, Seungtaek Choi, Pamela J. Schlembach, Sean E. McGuire, Quynh Nguyen, Steven J. Frank, Rajat J. Kudchadker, Weiliang Du, and Deborah A. Kuban, The University of Texas, MD Anderson Cancer Center, Houston; Andrew K. Lee, Texas Center for Proton Therapy, Irving, TX; K. Ranh Voong, The Johns Hopkins Hospital, Baltimore, MD; and Thomas J. Pugh, University of Colorado School of Medicine, Boulder, CO.

\section{Support}

Supported in part by the Cancer Center Support Grant (National Cancer Institute Grant No. P30 CA016672). 
Randomized Trial of Hypofractionated, Dose-Escalated, Intensity-Modulated Radiation Therapy (IMRT) Versus Conventionally Fractionated IMRT for Localized Prostate Cancer

The following represents disclosure information provided by authors of this manuscript. All relationships are considered compensated. Relationships are self-held unless noted. I = Immediate Family Member, Inst = My Institution. Relationships may not relate to the subject matter of this manuscript. For more information about ASCO's conflict of interest policy, please refer to www.asco.org/rwc or ascopubs.org/jco/site/ifc.

\section{Karen E. Hoffman}

No relationship to disclose

\section{K. Ranh Voong}

No relationship to disclose

\section{Lawrence B. Levy}

No relationship to disclose

Pamela K. Allen

No relationship to disclose

\section{Seungtaek Choi}

No relationship to disclose

Pamela J. Schlembach

No relationship to disclose

\section{Andrew K. Lee}

Employment: Texas Oncology

Sean E. McGuire

No relationship to disclose

\section{Quynh Nguyen}

No relationship to disclose

\section{Thomas J. Pugh}

Travel, Accommodations, Expenses: Augmenix

\section{Steven J. Frank}

Leadership: C4 Imaging

Stock or Other Ownership: C4 Imaging

Honoraria: Varian Medical Systems

Consulting or Advisory Role: Varian Medical Systems

Research Funding: Elekta

Patents, Royalties, Other Intellectual Property: C4 Imaging

Travel, Accommodations, Expenses: Varian Medical Systems

Rajat J. Kudchadker

No relationship to disclose

Weiliang Du

No relationship to disclose

Deborah A. Kuban

No relationship to disclose 


\section{Appendix}

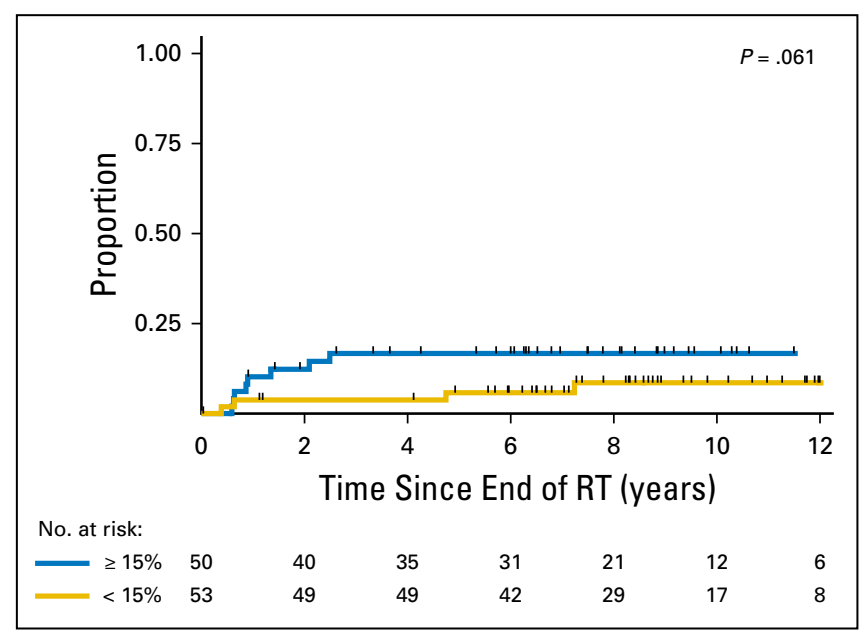

Fig A1. Grade 2 or 3 late GI toxicity in men who received HIMRT stratified by volume of rectum receiving $65 \mathrm{~Gy} \leq 15 \%$ versus $>15 \%$. HIMRT, hypofractionated intensity modulated radiation therapy; $\mathrm{RT}$, radiation therapy.

Table A1. Radiation Dose to the Rectum and Bladder in Men Who Received CIMRT or HIMRT and Assessed for Late Genitourinary and GI Toxicity

\begin{tabular}{|c|c|c|c|c|c|c|}
\hline \multirow[b]{2}{*}{ Organ } & \multicolumn{6}{|c|}{ No. of Patients Who Received Specified Dose to Given Percentage of Organ } \\
\hline & $<5 \%$ & $\begin{array}{l}5 \% \text { to } \\
<10 \%\end{array}$ & $\begin{array}{l}10 \% \text { to } \\
<15 \%\end{array}$ & $\begin{array}{l}15 \% \text { to } \\
<20 \%\end{array}$ & $\begin{array}{l}20 \% \text { to } \\
<25 \%\end{array}$ & $25 \%-30 \%$ \\
\hline \multicolumn{7}{|l|}{ Rectum } \\
\hline HIMRT, rectum received 65 Gy & 0 & 15 & 37 & 43 & 7 & 0 \\
\hline CIMRT, rectum received $70 \mathrm{~Gy}$ & 1 & 15 & 58 & 26 & 2 & 0 \\
\hline \multicolumn{7}{|l|}{ Bladder } \\
\hline HIMRT, bladder received 65 Gy & 10 & 51 & 29 & 8 & 3 & 1 \\
\hline CIMRT, bladder received $70 \mathrm{~Gy}$ & 16 & 38 & 30 & 11 & 5 & 2 \\
\hline
\end{tabular}

Abbreviations: CIMRT, conventional intensity-modulated radiation therapy; HIMRT, hypofractionated intensity-modulated radiation therapy. 
Hoffman et al

\begin{tabular}{|c|c|c|}
\hline \multicolumn{3}{|c|}{$\begin{array}{c}\text { Table A2. Frequency of Treatment Failure in Subgroups of Patients Who } \\
\text { Received CIMRT or HIMRT }\end{array}$} \\
\hline Subgroup & No. of Patients & $\begin{array}{l}\text { No. of Treatment } \\
\text { Failure Events }\end{array}$ \\
\hline \multicolumn{3}{|c|}{$P S A \leq 10 \mathrm{ng} / \mathrm{mL}(\mathrm{n}=186)$} \\
\hline CIMRT & 91 & 18 \\
\hline HIMRT & 95 & 9 \\
\hline \multicolumn{3}{|c|}{$\mathrm{PSA}>10 \mathrm{ng} / \mathrm{mL}(\mathrm{n}=20)$} \\
\hline CIMRT & 11 & 3 \\
\hline HIMRT & 9 & 1 \\
\hline \multicolumn{3}{|l|}{ No ADT $(n=156)$} \\
\hline CIMRT & 79 & 20 \\
\hline HIMRT & 77 & 9 \\
\hline \multicolumn{3}{|l|}{ Yes ADT $(n=50)$} \\
\hline CIMRT & 23 & 1 \\
\hline HIMRT & 27 & 1 \\
\hline \multicolumn{3}{|c|}{$\begin{array}{l}\text { Abbreviations: ADT, androgen deprivation therapy; CIMRT, conventional } \\
\text { intensity-modulated radiation therapy; HIMRT, hypofractionated intensity- } \\
\text { modulated radiation therapy; PSA, prostate-specific antigen. }\end{array}$} \\
\hline
\end{tabular}

Table A3. Cumulative Incidence of Treatment Failure in Subgroups of Patients Who Received CIMRT or HIMRT

\begin{tabular}{|c|c|c|c|c|c|c|}
\hline Subgroup & 5 Year & \multicolumn{4}{|c|}{ Cumulative Incidence of Failure, \% } & $95 \% \mathrm{Cl}$ \\
\hline \multicolumn{7}{|l|}{$\overline{\text { No ADT }}$} \\
\hline HIMRT & 9.5 & 4.7 to 19.0 & 12.7 & 6.8 to 23.0 & 12.7 & 6.8 to 23.0 \\
\hline \multicolumn{7}{|c|}{$\mathrm{PSA} \leq 10 \mathrm{ng} / \mathrm{mL}$} \\
\hline CIMRT & 8.2 & 4.0 to 164 & 14.8 & 8.3 to 25.6 & 24.4 & 14.9 to 38.3 \\
\hline HIMRT & 7.9 & 3.8 to 15.8 & 10.5 & 5.6 to 19.3 & 10.5 & 5.6 to 19.3 \\
\hline
\end{tabular}

Abbreviations: ADT, androgen deprivation therapy; CIMRT, conventional intensity-modulated radiation therapy; HIMRT, hypofractionated intensity-modulated radiation therapy; PSA, prostate-specific antigen. 